\title{
IDENTIFICACIÓN Y EVALUACIÓN DE LA INCIDENCIA DE INSECTOS Y HONGOS BENÉFICOS ASOCIADOS A Diaphorina citri KUWAYAMA (HEMIPTERA: LIVIIDAE) EN PLANTAS TRASPATIO (Citrus spp. y Murraya paniculata) DEL CANTÓN CATAMAYO (LOJA - ECUADOR)
}

\author{
Erráez Aguilera, Marlon ${ }^{a^{*}}$; Mazón, Marina ${ }^{a}$; Troya Armijos, Henry ${ }^{\mathbf{b}}$, Valarezo Espinoza, Doris ${ }^{\mathbf{b}}$ \\ ${ }^{a}$ Universidad Nacional de Loja, Carrera de Ingeniería Agronómica, Avenida Pio Jaramillo \\ Alvarado, Loja, Ecuador. \\ ${ }^{\mathrm{b}}$ Agencia de Regulación y Control Fito y Zoosanitario, Laboratorio Regional, Av. Turunuma y \\ Cádiz / Loja, Ecuador.
}

Ingresado: 25/11/2019

\section{Resumen}

En los últimos años, la citricultura mundial se ha encontrado amenazada por la presencia del psílido asiático de los cítricos, Diaphorina citri, un insecto perjudicial por ser portador de la bacteria "Candidatus Liberibacter asiaticus (las)", causante de la enfermedad Huanglongbing (HLB), que ha provocado la devastación de plantaciones y la reducción de la producción de cítricos en todo el mundo. En el año 2017 se reportó la presencia de D. citri en plantas traspatio del cantón Catamayo, en la provincia de Loja, por lo que la presente investigación tuvo por objetivo identificar los enemigos naturales (depredadores, parasitoides y hongos) asociados a D. citri, y evaluar su incidencia en campo. Para ello, se realizaron muestreos en plantas traspatio (Citrus spp. y Murraya paniculata) del cantón Catamayo. Los muestreos se efectuaron a una altura de $1,5 \mathrm{~m}$ de las plantas en los cuatro puntos cardinales de la misma. Se colectaron larvas y adultos de depredadores, ninfas parasitadas y adultos de parasitoides, y adultos de D. citri para el aislamiento de hongos. Como resultado de la identificación en el laboratorio, se determinó la presencia de 11 especies depredadoras (principalmente Cheilomenes sexmaculata y Chrysopa spp.), dos especies de parasitoides (Diaphorencyrtus sp. y Tamarixia radiata) y cuatro

\footnotetext{
* Correspondencia a: Universidad Nacional de Loja, Carrera de Ingeniería Agronómica, Avenida Pio Jaramillo Alvarado, Loja, Ecuador. Teléfono: +593985992105. Correo electrónico: erraezmarlon@gmail.com
}

Aceptado: 22/04/2020

especies de hongos (Cladosporium sp., Fusarium sp., Aspergillus sp. y Alternaria sp.). Diaphorencyrtus sp. tuvo una tasa de parasitismo del 7,76\%. Estas especies podrían utilizarse para establecer programas de control biológico, aprovechando su potencial como reguladores de poblaciones de ninfas de Diaphorina citri.

Palabras clave: Depredadores, Diaphorina citri, enemigos naturales, hongos entomopatógenos, parasitoides.

IDENTIFICATION AND EVALUATION OF THE INCIDENCE OF INSECTS AND BENEFICIAL FUNGI ASSOCIATED TO Diaphorina citri KUWAYAMA (HEMIPTERA: LIVIIDAE) IN BACKYARD PLANTS (Citrus spp. and Murraya paniculata) AT CATAMAYO CANTON (LOJA - ECUADOR)
Abstract
In recent years, the global citrus industry has been threatened by the presence of the Asian citrus psyllid, Diaphorina citri, a harmful insect which carriers the "Candidatus Liberibacter asiaticus (las)", a bacterium that causes the Huanglongbing disease (HLB), which has led to the devastation of plantations and reduction of citrus production worldwide. In 2017, the presence of D. citri was reported in backyard plants of Catamayo canton, in Loja province; therefore, the aim of this research was to identify the natural enemies (predators, parasitoids and fungi) associated with $D$. citri, and 
to evaluate their incidence in the backyard plants (Citrus spp. and Murraya paniculata) at Catamayo canton. The samples were taken at 1,5 m height of the four cardinal points of the plants. Larvae and adults of predators, parasitized nymphs and adult parasitoids, and adults of $D$. citri were collected. A total of 11 predatory species were identified (mainly Cheilomenes sexmaculata and Chrysopa spp.), two species of parasitoids (Diaphorencyrtus sp. and Tamarixia radiata) and four species of fungi (Cladosporium sp., Fusarium sp., Aspergillus sp. and Alternaria sp.). Diaphorencyrtus sp. showed a parasitism rate of $7,76 \%$. These species could be used to establish biological control programs, taking advantage of their potential as regulators of nymph populations of Diaphorina citri.

Keywords: Diaphorina citri, entomopathogenic fungi, natural enemies, parasitoids, predators.

\section{INTRODUCCIÓN}

Los cítricos son frutas valoradas por sus propiedades nutricionales, en especial por su alto contenido de ácido ascórbico (vitamina C). Presentan una producción a nivel mundial de más de 70 millones de toneladas [1], que supera a la de todas las frutas de hoja caduca, tales como: manzanas, peras, melocotones, ciruelas, etc., abarcando el $20 \%$ del mercado mundial de frutas [2].

En Ecuador, la producción de cítricos en los últimos años se ha incrementado sustancialmente, aportando en gran medida a la economía del país [3]. La superficie cultivada es de aproximadamente 22 mil hectáreas, con una producción de 104 mil toneladas, siendo la naranja y el limón los cítricos más representativos [4].

En la provincia de Loja, la naranja se cultiva en un área de 75 ha y el limón en 85 ha, de las que se obtiene una producción anual de 203 y 896 toneladas respectivamente [4]. En el cantón Catamayo, el limón aporta en gran parte a la economía familiar, puesto que se cultivan 23 ha, obteniéndose una producción aproximada de 166,32 toneladas $(29,85 \%$ de rentabilidad) [5].

Una gran limitante en la producción de cítricos es la alta exposición de estos a numerosos organismos fitófagos y fitopatógenos, los cuales causan pérdidas económicas en plantaciones en todo el mundo [6]. En los últimos años se ha centrado la atención en el psílido asiático de los cítricos, Diaphorina citri Kuwayama (Hemiptera:
Liviidae), un fitopatógeno que constituye una de las plagas más destructoras de la citricultura a nivel mundial $[7,8,9]$. Tanto las ninfas como los adultos de esta especie causan cuantiosos daños en los cítricos, ya que extraen la savia y debilitan a la planta, a la vez que inyectan toxinas que inducen al enrollamiento de las hojas, impidiendo así el crecimiento normal y produciendo malformaciones foliares [10]. Sin embargo, el daño más importante que causa es la trasmisión de la bacteria "Candidatus Liberibacter asiaticus (las)", causante de la enfermedad comúnmente conocida como "Huanglongbing" (HLB), considerada como la más devastadora de plantaciones citrícolas [11].

D. citri fue reportada por primera vez en el Ecuador en el año 2014, en la ciudad de Guayaquil, en plantas de Murraya exotica encontradas a lo largo de una acera [12]. En el año 2017, en el cantón Catamayo se encontraron plantas de cítricos atacadas por D. citri; sin embargo, aún no se ha registrado la bacteria causante del HLB [13].

Lo expuesto podría sugerir que la citricultura en este cantón podría verse amenazada por la presencia del psílido asiático, por lo que este requeriría ser controlado antes de su diseminación a huertos citrícolas. Con el objetivo de identificar enemigos naturales (insectos y hongos entomopatógenos) de Diaphorina citri y evaluar su incidencia natural en plantas traspatio (Citrus spp. y Murraya paniculata) del cantón Catamayo se realizó esta investigación, a fin de desarrollar estrategias de control biológico.

\section{METODOLOGÍA}

La presente investigación se realizó en la zona urbana del cantón Catamayo, que se encuentra a $38 \mathrm{~km}$ al noroeste de la provincia de Loja. La ubicación se seleccionó en base en los dos puntos donde se constató por primera vez la presencia de D. citri en este cantón [13], de acuerdo a las siguientes coordenadas: $9558488 \mathrm{~m} \mathrm{~S}, 682225 \mathrm{~m}$ E y 9560457 m S, 680714 m E. Los muestreos se realizaron durante el período noviembre 2018 febrero 2019, de preferencia en plantas jóvenes traspatio (< 4 años) de mirto (Murraya paniculata) y cítricos (Citrus spp.), en los sectores Buenavista, Cementerio, Parque $1^{\text {ro }}$ de Mayo, Entrada a Trapichillo, Trapichillo y Guayabal, cada 10 - 15 días durante dos meses (cuatro muestreos totales), en horas de la mañana (7:00 a 10:00). En cada muestreo se inspeccionaron aproximadamente 40 plantas traspatio de los sectores antes mencionados 
a una altura aproximada de 1,5 m (estrato medio) en cada punto cardinal de la planta.

\section{Colecta e identificación de insectos depredadores y parasitoides}

Depredadores. Se realizaron inspecciones visuales en un área foliar de aproximadamente 20 $\mathrm{cm}^{3}$, donde se presenciaron poblaciones de ninfas de D. citri. Se colectaron insectos depredadores (inmaduros o adultos) que se encontraban dentro del área delimitada, ya sea que estuvieran atacando activamente o cerca de la población de ninfas. Los adultos se colocaron en frascos con alcohol al $70 \%$ y fueron llevados al Laboratorio Regional de Entomología de la Agencia de Regulación y Control Fito y Zoosanitario, localizado en la provincia de Loja, en donde se realizó su identificación utilizando un estéreo-microscopio Olympus SZX16 y claves ilustradas de géneros de Coccinellidae de América del Sur [14]. En el caso de larvas, estas se mantuvieron en cajas petri y fueron alimentadas con pulgones y ninfas de D. citri, hasta la emergencia de los adultos para su identificación.

Parasitoides. En cada rama donde se observó presencia de ninfas de $D$. citri, se colectó un brote tierno de $7 \mathrm{~cm}$ a $10 \mathrm{~cm}$ de longitud, se colocó en una funda de papel y se trasladó al Laboratorio Regional de Entomología de la Agencia.

En el laboratorio se aplicó el método usado por Hernández [15], que consistió en cuantificar el número de ninfas de cada brote. Se colocaron las muestras en vasos desechables de plástico con algodón humedecido para mantener el brote fresco y la humedad adecuada. Los vasos se cubrieron con tela de polipropileno sujeta con una liga para evitar el escape de los adultos del psílido o de avispitas parasitoides emergidas de las ninfas, así como para evitar que se introdujeran otros insectos. Se evaluaron de preferencia ninfas de penúltimo y último instar (N4 y N5) de D. citri [16].

En esas condiciones se mantuvieron las muestras hasta la emergencia de los adultos, ya fuera de parasitoides o de D. citri. Posteriormente, se colocaron los adultos de los parasitoides en alcohol al $70 \%$ y se identificaron haciendo uso de un estéreo-microscopio Olympus SZX16 y mediante claves taxonómicas $[17,18,19]$.

\section{Colecta e identificación de hongos entomopatógenos}

En las mismas ramas donde se buscaron los depredadores se colectaron adultos muertos de
D. citri con síntomas de infección por hongos entomopatógenos [20]. Al no encontrar adultos infectados con hongos, se procedió a colectar ejemplares aparentemente sanos para buscar esporas viables, los cuales se colocaron en bolsas individuales de papel y se llevaron al Laboratorio Regional de Fitopatología de la Agencia, localizado en Loja.

Se realizó el aislamiento de las esporas siguiendo el procedimiento del laboratorio [21], el cual consistió en desinfectar el ejemplar con cloro al 2\% y colocarlo en medio PDA a $26^{\circ} \mathrm{C}$ y cámara húmeda. Luego de 8 días, se colocó parte del micelio en un portaobjetos para la identificación mediante claves taxonómicas especializadas [22], haciendo uso de un microscopio Boeco Bm120.

\section{Evaluación de la incidencia de enemigos naturales en campo}

La evaluación del porcentaje de incidencia se realizó únicamente para insectos parasitoides y hongos entomopatógenos, puesto que para evaluar la incidencia de insectos depredadores en campo, necesariamente se debe encontrar al insecto atacando poblaciones de ninfas, lo cual era muy poco probable.

En el caso de los parasitoides, se cuantificaron las ninfas no parasitadas y las parasitadas (parasitadas y momificadas), y se calculó el porcentaje de parasitismo con la siguiente ecuación [23, 24]:

Ec.1.

$\%$ de parasitismo $=\frac{\text { Ninfas Parasitadas }}{\text { Total de Ninfas muestreadas }} * 100$

Para evaluar la incidencia de los hongos entomopatógenos se cuantificó el número total de adultos vivos y muertos, y se calculó con la siguiente ecuación [21]:

Ec.2.

Incidencia de hongos $=\frac{\text { Adultos muertos }}{\text { Total de Adultos muestreados }} * 100$

\section{RESULTADOS Y DISCUSIÓN}

A lo largo de todo el muestreo se capturó un total de 13 especies en las localidades de: Buenavista, Cementerio, Parque $1^{\text {ro }}$ de Mayo, Entrada a Trapichillo, Trapichillo y el Guayabal, en plantas traspatio de Murraya paniculata y Citrus spp. de la zona urbana del cantón Catamayo (Tabla 1). 
Tabla 1: Ninfas de Diaphorina citri y enemigos naturales registrados en el cantón Catamayo durante el periodo de muestreo noviembre 2018 - febrero 2019.

\begin{tabular}{|c|c|c|c|c|c|c|c|}
\hline \multirow{2}{*}{$\begin{array}{l}\text { Ninfas D. citri y } \\
\text { enemigos naturales }\end{array}$} & \multicolumn{7}{|c|}{ Número de especímenes en cada localidad } \\
\hline & Buenavista & Cementerio & $\underset{\text { Mayo }}{\text { Parque } 1^{\text {ro }} \text { de }}$ & $\begin{array}{c}\text { Entrada a } \\
\text { Trapichillo }\end{array}$ & Trapichillo & Guayabal & Total \\
\hline $\begin{array}{l}\text { Ninfas de Diaphorina } \\
\text { citri (Kuwayama) }\end{array}$ & 103 & 10 & 28 & 10 & 72 & 69 & 292 \\
\hline \multicolumn{8}{|l|}{ Depredadores } \\
\hline Azya orbigera ecuadorica & 0 & 0 & 0 & 2 & 0 & 0 & 2 \\
\hline Cheilomenes sexmaculata & 2 & 0 & 1 & 2 & 3 & 3 & 9 \\
\hline Hyperaspis esmeralda & 3 & 0 & 0 & 0 & 0 & 0 & 3 \\
\hline Hippodamia convergens & 0 & 1 & 1 & 0 & 0 & 0 & 2 \\
\hline Paraneda pallidula & 1 & 0 & 0 & 0 & 0 & 0 & 1 \\
\hline Pentilia sp. & 1 & 0 & 0 & 0 & 0 & 0 & 1 \\
\hline Rodolia cardinalis & 1 & 0 & 0 & 0 & 0 & 0 & 1 \\
\hline Hyperaspis onerata & 1 & 0 & 1 & 0 & 1 & 0 & 3 \\
\hline Diomus sp. & 2 & 0 & 0 & 0 & 0 & 0 & 2 \\
\hline Chrysopa spp. & 4 & 0 & 0 & 0 & 1 & 0 & 5 \\
\hline Orius spp. & 1 & 1 & 0 & 0 & 0 & 0 & 2 \\
\hline \multicolumn{8}{|l|}{ Parasitoides } \\
\hline Diaphorencyrtus sp. & 21 & 0 & 2 & 0 & 0 & 0 & 23 \\
\hline Tamarixia radiata & 1 & 0 & 0 & 0 & 0 & 1 & 2 \\
\hline TOTAL & 38 & 2 & 5 & 4 & 5 & 2 & 56 \\
\hline
\end{tabular}

De las 13 especies encontradas, 11 fueron depredadores y dos (2) parasitoides (Fig. 1). Entre los depredadores, se encontraron 9 especies pertenecientes a la familia Coccinellidae (Coleoptera), una a la familia Chrysopidae (Neuroptera) y una a la familia Anthocoridae (Hemiptera).

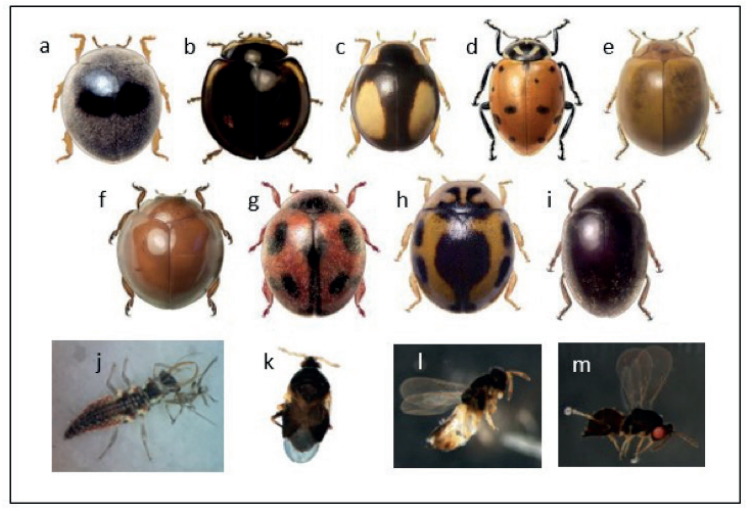

Fig. 1. Enemigos naturales asociados con Diaphorina citri en Catamayo. a. Azya orbigera b. Cheilomenes sexmaculata c. Hyperaspis esmeralda d. Hippodamia convergens e. Paraneda palludula f. Pentilia sp. g. Rodolia Cardinalis h. Hyperaspis onerata i. Diomus sp. (Fuente: González [14]) j. Chrysopa sp. k. Orius sp. l. Diaphorencyrtus sp. m. Tamarixia radiata.
Como parasitoides, se encontraron dos (2) especies de las familias Encyrtidae y Eulophidae respectivamente (Hymenoptera). Las especies encontradas se encuentran entre los 101 artrópodos reportados en el mundo como enemigos naturales de D. citri $[25,26,27]$.

La mayor población de ninfas de D. citri se encontró en el sector Buenavista, correspondiente a 103 ninfas de un total de 292 ninfas obtenidas durante la evaluación de brotes en laboratorio, $\mathrm{y}$ en consecuencia fue en este sector donde se encontró la mayor cantidad de especies benéficas colectadas. Del total de ninfas confinadas, el $20 \%$ murieron por causas desconocidas.

\section{Depredadores}

En el transcurso de los muestreos, se constató la presencia de depredadores en distintos estados de desarrollo cerca de poblaciones de ninfas de $D$. citri, y en algunos casos, incluso se evidenció el ataque directo de los depredadores sobre dichas poblaciones. En los sectores Buenavista, Cementerio, Parque $1^{\text {ro }}$ de Mayo, Entrada a Trapichillo y Guayabal se encontraron 
depredadores en estado adulto, posturas de huevos (Fig. 2a) y larvas de Coccinélidos (Fig. 2b y Fig. 2c) que estaban cerca de poblaciones de $D$. citri. En los sectores Buenavista y Guayabal se encontraron posturas (Fig. 2d) y larvas (Fig. 2e) de Chrysopa sp. En el laboratorio se evidenció el ataque de larvas de Coccinélidos (Fig. 2f) y Chrysopa sp. (Fig. 2g) sobre ninfas de $D$. citri.

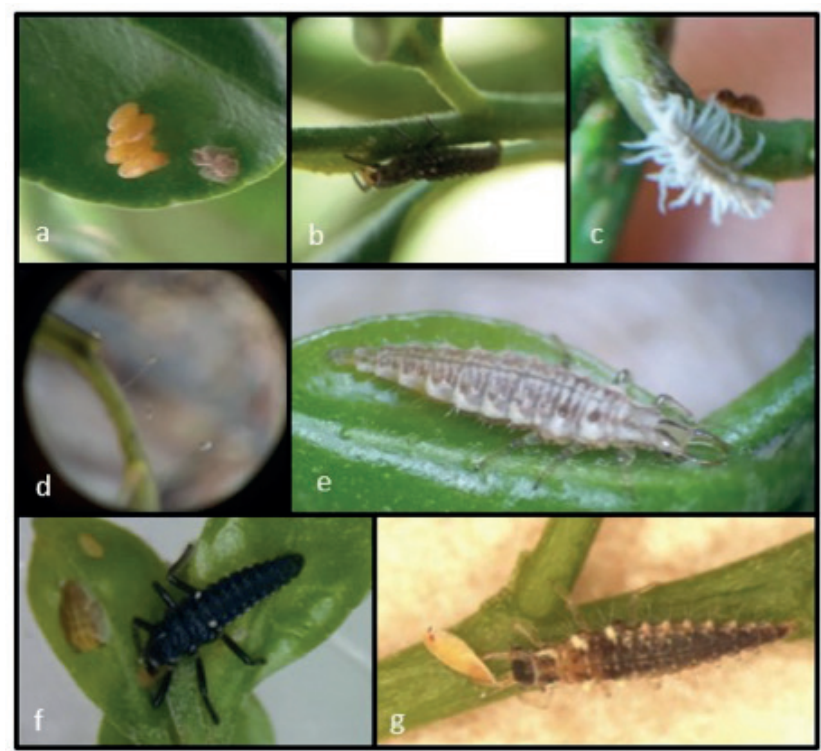

Fig. 2. Depredadores de D. citri. a. Posturas de Coccinélidos. b y c. Larvas de Coccinélidos. d. Posturas de Chrysopa sp. e. Larva de Chrysopa sp. f. Larva de Coccinélido atacando ninfa de D. citri. g. Larva de Chrysopa sp. atacando ninfa de D. citri.

Diversos estudios en Colombia [28] y en México $[29,30,31,32]$ reportan las especies aquí encontradas como depredadores y de uso potencial para el control biológico de D. citri. De las especies identificadas, la más frecuente fue Cheilomenes sexmaculata, especie reportada en Guayaquil, que se encontró alimentándose de ninfas de $D$. citri [23], considerada como uno de los depredadores más efectivos para el control de poblaciones de ninfas de D. citri [33]. Otras especies encontradas cerca de poblaciones de ninfas de D. citri y que poseen una alta incidencia en su control son las pertenecientes al género Chrysopa [33,34,35]. $\mathrm{Su}$ eficiencia depredadora se constató en el laboratorio al colocar diez pulgones y una ninfa de D. citri para su alimentación; se observó que, a pesar de la abundancia de pulgones, Chrysopa sp. optó por alimentarse de la ninfa de D. citri.

\section{Parasitoides}

Durante los muestreos se encontraron ninfas de $D$. citri parasitadas (momificadas) (Fig. 3a) y ninfas momificadas vacías con el orificio por donde emergen los insectos parasitoides (Fig. 3b). Una vez que se realizó el seguimiento de los brotes en el laboratorio, se presenció la emergencia de los adultos parasitoides correspondientes a Tamarixia radiata (Fig. 3c) y Diaphorencyrtus sp. (Fig. 3d). Una característica muy general de los demás artrópodos colectados (depredadores) es que son generalistas, es decir, que no solo se alimentan de ninfas de $D$. citri, a diferencia del ectoparasitoide, Tamarixia radiata, y el endoparasitoide, Diaphorencyrtus aligarhensis, que se han reportado como parasitoides primarios específicos de ninfas de D. citri. $[25,26,27,24,19,18,36]$.

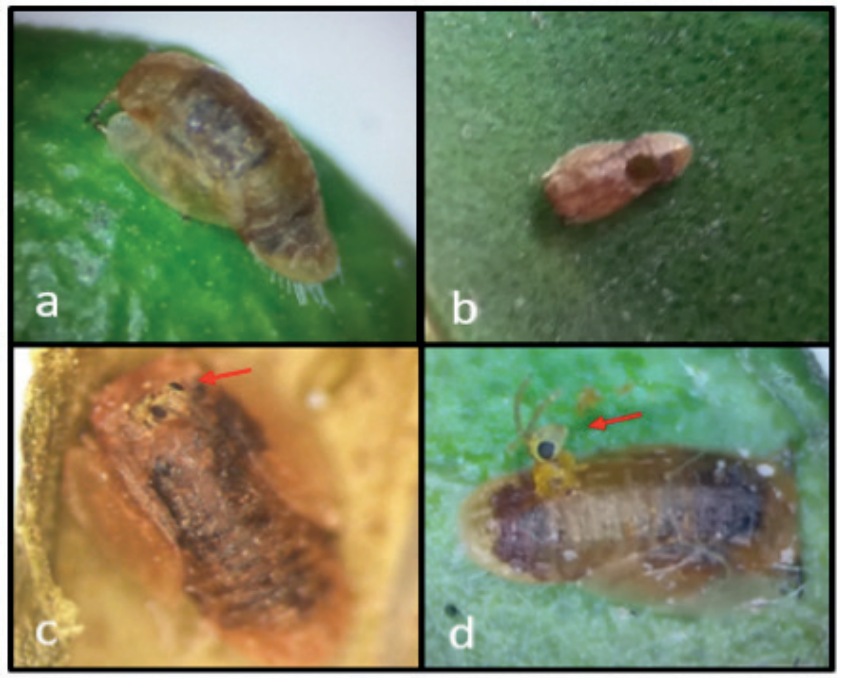

Fig. 3. Constatación de la presencia de parasitoides en los muestreos: a. Ninfa momificada de Diaphorina citri. b. Ninfa momificada con orificio de emergencia del parasitoide Diaphorencyrtus sp. c. Adulto de Tamarixia radiata (flecha) emergiendo de la ninfa de D. citri. d. Adulto de Diaphorencyrtus sp. (flecha) emergiendo de la ninfa de D. citri.

\section{Hongos entomopatógenos asociados a Diaphorina citri}

Durante los muestreos, no se encontró actividad de hongos entomopatógenos en campo (adultos muertos micosados); sin embargo, a partir de 95 adultos vivos colectados en el sector Buenavista, donde se consiguió la mayor población de D. citri, se obtuvieron varias colonias de hongos: 48 adultos desarrollaron Cladosporium sp., uno desarrolló Fusarium sp., uno (1) Aspergillus sp. y cinco (5) adultos desarrollaron Alternaria sp. (Fig. 4).

La falta de humedad del sector podría ser la causa por la cual no se encontraron ninfas ni adultos micosados en campo, ya que el sector cuenta con un clima muy seco y las plantas traspatio no disponen de riego ni de sombra, que les permita mantener una humedad óptima (81\% - $92 \%)$ para el desarrollo de hongos entomopatógenos [37]. 


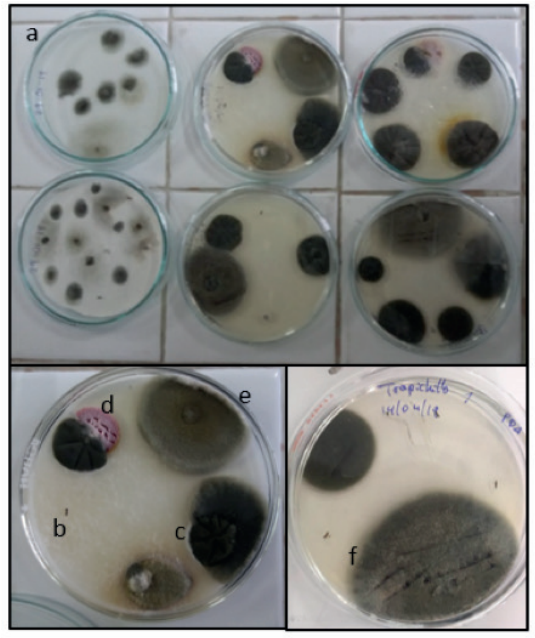

Fig. 4. Resultados del aislamiento de adultos de Diaphorina citri en PDA y cámara húmeda. a. Colonias de hongos. b. Adulto sin micosamiento. c. Colonia de Cladosporium sp. d. Colonia de Fusarium sp. e. Colonia de Aspergillus sp. f. Colonia de Alternaria sp.

\section{Incidencia de los enemigos naturales en campo}

La evaluación de la incidencia de depredadores en campo se dificultó debido a que no se encontraron todos los especímenes colectados atacando poblaciones de ninfas. Algunos se encontraron cerca a estas, y por medio de literatura y estudios realizados [28] se corroboró que son enemigos naturales de $D$. citri.

Respecto a la incidencia en campo de parasitoides, solo se calculó el porcentaje de parasitismo para el sector Buenavista, debido a que solo en dicho sector se encontraron ninfas con síntomas de parasitismo, de las cuales posteriormente se obtuvieron los adultos en laboratorio.
En el caso de Tamarixia radiata, su tasa de parasitismo puede ser muy variable $(0 \%$ a $80 \%)$ $[23,28,29,30,38,39]$ debido a diversos factores, tales como: condiciones ambientales favorables, uso de insecticidas o presencia de organismos benéficos, entre otros, registrándose las mayores tasas de parasitismo a $26,3{ }^{\circ} \mathrm{C}$ [36], por lo que esta especie podría ser eficiente para controlar poblaciones de D. citri en el cantón Catamayo si se incrementara su población. Sin embargo, en el presente estudio solo dos ejemplares estaban parasitados por esta especie.

La especie Diaphorencyrtus sp. se encontró en mayor cantidad en Buenavista, sector donde se detectó el primer foco infeccioso de la plaga y donde se presenció la mayor población de ninfas de D. citri. En otros sectores no se detectó la presencia de parasitoides, probablemente por las aplicaciones de insecticidas que se habían realizado, según indicaron moradores del sector, ya que estos provocan la disminución considerable de la fauna benéfica (parasitoides y depredadores) [40].

La tasa de parasitismo de Diaphorencyrtus sp. fue del $7,76 \%$ (Tabla 2 ), resultado similar $(6,3 \%)$ al obtenido en México [30] bajo condiciones similares. La baja tasa de parasitismo de Diaphorencyrtus sp. podría deberse a la presencia de otros insectos benéficos como es el caso de los depredadores, que pueden llegar a alimentarse de las ninfas ya parasitadas antes de llegar a momificarse, y al uso de insecticidas que además de reducir la cantidad de fauna benéfica, reduce las de poblaciones de ninfas de $D$. citri, y en consecuencia la reproducción de Diaphorencyrtus sp. [41].

Tabla 2: Número de ninfas de D. citri colectadas y porcentaje de parasitismo durante el periodo de muestreo noviembre 2018 - febrero 2019.

\begin{tabular}{|c|c|c|c|c|c|c|c|}
\hline Sector & $\begin{array}{c}M 1 \\
09 / 11 / 2018\end{array}$ & $\begin{array}{c}M 2 \\
22 / 11 / 2018\end{array}$ & $\begin{array}{c}\text { M3 } \\
17 / 12 / 2018\end{array}$ & $\begin{array}{c}M 4 \\
24 / 01 / 2019\end{array}$ & $\begin{array}{c}\text { Total } \\
\text { de ninfas }\end{array}$ & $\begin{array}{c}\text { Ninfas } \\
\text { parasitadas }\end{array}$ & $\begin{array}{c}\text { Tasa de } \\
\text { parasitismo } \\
\text { (Diaphorencyrtus sp.) }\end{array}$ \\
\hline Buenavista & 46 & 30 & 17 & 10 & 103 & 8 & $7,76 \%$ \\
\hline Cementerio & 10 & 0 & 0 & 0 & 10 & 0 & 0 \\
\hline $\begin{array}{l}\text { Parque 1ro } \\
\text { de Mayo }\end{array}$ & 16 & 7 & 5 & 0 & 28 & 0 & 0 \\
\hline $\begin{array}{l}\text { Entrada a } \\
\text { Trapichillo }\end{array}$ & 10 & 0 & 0 & 0 & 10 & 0 & 0 \\
\hline Guayabal & 34 & 19 & 16 & 0 & 69 & 0 & 0 \\
\hline Trapichillo & 37 & 21 & 14 & 0 & 72 & 0 & 0 \\
\hline
\end{tabular}


En lo que respecta a la incidencia de los hongos entomopatógenos, el $43,16 \%$ de los adultos muestreados no desarrolló ninguna colonia de hongo, por lo que se obtuvo un porcentaje de incidencia del 56,84\%, del cual, el $49,47 \%$ fue por Cladosporium sp., el 1,05\% de Fusarium sp., el $1,05 \%$ de Aspergillus sp. y el 5,26\% de Alternaria sp. (Tabla 3). Los géneros de hongos aislados a partir de adultos vivos de D. citri corresponden a varias cepas reportadas anteriormente por diversos autores [26].
De estas, Fusarium y Cladosporium podrían ser útiles para la elaboración de bioplaguicidas para el control de D. citri; en cambio, Aspergillus y Alternaria no han sido reportados como enemigos naturales de D. citri $[26,42,43]$ por lo que se podrían hacer bioensayos de patogenicidad para evaluar si es posible usarlas como controladores de poblaciones de la plaga. Sin embargo, estas especies no son lo suficientemente agresivas como para matar al insecto, debido a que los ejemplares de donde se los obtuvo estaban vivos y sanos.

Tabla 3: Porcentaje de incidencia de hongos obtenidos a partir del aislamiento de adultos de Diaphorina citri.

\begin{tabular}{lcccccccc}
\hline & Buenavista & Cementerio & $\begin{array}{c}\text { Parque 1 } \\
\text { Mayo }\end{array}$ & $\begin{array}{c}\text { Entradaa } \\
\text { Trapichillo }\end{array}$ & Trapichillo & Guayabal & Total & $\begin{array}{c}\text { Tasa de } \\
\text { Infestación }\end{array}$ \\
\hline Sin colonia & 6 & 0 & 3 & 0 & 32 & 0 & 41 & $43,16 \%$ \\
Cladosporium sp. & 35 & 0 & 8 & 0 & 4 & 0 & 47 & $49,47 \%$ \\
Fusarium sp. & 1 & 0 & 0 & 0 & 0 & 0 & 1 & $1,05 \%$ \\
Aspergillus sp. & 1 & 0 & 0 & 0 & 0 & 0 & 1 & $1,05 \%$ \\
Alternaria sp. & $\mathbf{0}$ & 0 & 3 & 0 & 2 & 0 & 5 & $5,26 \%$ \\
Total & $\mathbf{4 3}$ & $\mathbf{0}$ & $\mathbf{1 4}$ & $\mathbf{0}$ & $\mathbf{3 8}$ & $\mathbf{0}$ & $\mathbf{9 5}$ & $\mathbf{1 0 0 \%}$ \\
\hline
\end{tabular}

Las especies encontradas en el cantón Catamayo, por su eficiencia como controladores de D. citri, podrían representar una solución para controlar poblaciones de la plaga, reduciendo la dependencia de insecticidas que contaminan a otros elementos bióticos (depredadores, parasitoides, polinizadores, animales, plantas, hombre) y sistemas abióticos (suelo, agua y aire), amenazando de esta manera su estabilidad [6]. Por otra parte, es menester mencionar que los residuos de los insecticidas afectan a la salud humana, produciendo cáncer, daños al sistema reproductivo, al hígado, al cerebro y a otras partes del cuerpo [44], llegando a provocar la muerte de aproximadamente 200.000 personas cada año [45].

\section{CONCLUSIONES}

- En plantas traspatio del cantón Catamayo se colectaron e identificaron especies depredadoras y parasitoides de ninfas de $D$. citri, las mismas que ya se han registrado como potenciales controladores de ninfas.

- Los géneros de hongos obtenidos en esta investigación fueron aislados a partir de adultos vivos de $D$. citri, debido a que no se encontraron adultos micosados en campo, por lo que es necesario realizar evaluaciones mediante bioensayos para corroborar si son entomopatógenos y para determinar su patogenicidad en los distintos estados de desarrollo del insecto.

- El porcentaje de incidencia de los organismos benéficos encontrados durante esta investigación y su frecuencia indican que podrían ser buenos para establecerse como agentes controladores de poblaciones de D. citri.

\section{REFERENCIAS}

[1] Zilch R. J. IX Seminario Internacional de Cítricos. Huanglongbing y su situación mundial. Lima - Perú. 2016.

[2] Lerma Carbajal J., Ibarra Tamez A. R., Galván González V., Martínez de la Cerda J., Rodríguez Rodríguez G. A., Tellez González V., Galicia Cavazos J. P. Situación de la Citricultura en Nueva León. Nueva León - México. 2015.

[3] Yépez A. B. Boletín Situacional de Naranja / MAGAP. Quito - Ecuador. 2015.

[4] Salazar D., Villafuerte W., Cuichán M., Orbe D., Márquez J. Quito - Ecuador: ESPAC. [Internet]. 2016 Disponible en: https://www.ecuadorencifras. gob.ec/documentos/web-inec/Estadisticas_ agropecuarias/espac/espac $2016 /$ Indice $\% 20$ de $\% 20$ publicacion\%20ESPAC\%202016.xlsx.

[5] Torres J. L. Análisis de la producción, 
comercialización y rentabilidad de Limón en el Cantón Catamayo. Tesis previa de Grado de Ing. en Administración y Producción Agropecuaria. Universidad Nacional de Loja - Ecuador. 2012.

[6] Rodríguez Quibrera C. G., Mendoza Herrera A. Una amenaza para la citricultura mexicana. La Ciencia y el Hombre. [Internet]. 2014; 8 (2). Disponible en: https://www.uv.mx/cienciahombre/ revistae/vol27num1/articulos/amenaza-para-lacitricultura.html.

[7] Hernández F. L. M., López J. I., Velázquez M J. J. Urías L. M. A., Gómez J. R., Robles B. A. Eficacia biológica de compuestos químicos de bajo impacto ambiental aplicados al suelo y follaje contra Diaphorina citri Kuwayama (Hemiptera: Psyllidae) en Citrus latifolia Tanaka. Revista Mexicana de Ciencias Agrícolas. ISSN 2007-0934. 2013; 4 (5): 687-7900.

[8] Salcedo B.D., Hinojosa R.A., Mora A.G., Covarrubias G.I., De Paolis F.J.J., Mora F.S., y Cíntora G.C. Evaluación del impacto económico de Huanglongbing (HLB) en la cadena citrícola mexicana. IICA. 2010; 144. DOI. 10.13140/RG.2.1.2123.0487.

[9] Pourreza, A., Lee,W. S., Ehsani R., Schueller J. K., Raveh E. An optimum method for real-time in-field detection of Huanglongbing disease using a vision sensor. Computers and Electronics in Agriculture. 2015; 110: 221-232.

[10] Lellani B. H., Aleman J., Martínez M. A., Suris M., Ravelo, J. Ciclo de vida horizontal de Diaphorina citri Kuwayama (Hemiptera: Psilidae) sobre Muralla paniculata L. Rev. Prot. Veg. 2012; 27.

[11] Gottwald T.R. Current epidemiological understanding of citrus huanglongbing. Annu. Rev. Phytopathol. 2010; 48: 119-139.

[12] Cornejo J.F., Chica E.J. First Record of Diaphorina citri (Hemiptera: Psyllidae) in Ecuador Infesting Urban Citrus and Orange Jasmine Trees. J. Insect Sci. 2014; 14 (298): 1-3.

[13] Pineda M. Foro: Retos y desafíos para el desarrollo agrícola de la provincia de Loja. Universidad Nacional de Loja. Loja - Ecuador; 2018. [14] González G. Los Coccinellidae de Perú [Internet]. 2007. Disponible en: http://www. coccinellidae.cl/paginasWebPeru/Paginas / InicioPeru.php.

[15] Hernández R. M. Inventario de Enemigos Naturales asociados a Diaphorina citri (Kuwayama, 1907) (Hemiptera: Psyllidae), en dos fincas de producción citrícola en el departamento de San Salvador, El Salvador. El Salvador. 2016.

[16] Barrera J. F., Herrera J., Gómez J., Valle, J. Muestreo de Diaphorina citri Kuwayama (Hemiptera: Psyllidae) en brotes de Limón. México. 2012.

[17] Zamora Mejías D., Hanson P. Clave dicotómica para especies parasitoides e hiperparasitoides (Hymenoptera) de áfidos (Hemiptera: Aphididae) de Costa Rica. 2017; 28(3): 565-575.
[18] Arias Ortega P.L., Restrepo García A.M., Soto Giraldo A. Primer registro de Diaphorencyrtus sp. (Hymenoptera: Encyrtidae) en Colombia. Bol. Cient. Mus. Hist. Nat. U. de Caldas. 2016; 20 (1): 157-165.

[19] Restrepo-García A.M., Arias-Ortega P.L., Soto-Giraldo A. Primer reporte de Tamarixia radiata (Waterston) (Hymenoptera: Eulophidae) en el departamento de Caldas, Colombia. Bol. Cient. Mus. Hist. Nat. U. de Caldas, 2016; 20 (1): 204-210.

[20] Ramos D. Y. Identificación de hongos entomopatógenos de Diaphorina citri Kuwayama, en los departamentos del Valle del Cauca y Quindío, Colombia. Palmira - Colombia. 2016.

[21] Agencia de Regulación y Control Fito y Zoosanitario. Procedimiento PEE/FP/07 Aislamiento e Identificación de Hongos. Laboratorio de Fitopatología. Loja - Ecuador. 2018.

[22] Barnett H. L., Hunter, B. B. Illustrated Genera of Imperfect Fungi. U.S.A: APS PRESS.1998

[23] Chavez Y., Chirinos D. T., González G., Lemos N., Fuentes A., Castro R., Kondo T. Tamarixia radiata (Waterston) and Cheilomenes sexmaculata (Fabricius) as biological control agents of Diaphorina citri Kuwayama in Ecuador. Guayaquil - Ecuador: Chilean Journal of Agricultural Research: 2017; $77(2)$.

[24] Sánchez González J., Vizcarra Valdez N., Moreno Carrillo G., Alpizar Puente R., Contreras Bermúdez Y., Sandoval Rodríguez D., Cordoba Urtiz, E. Manual de reproducción masiva de Tamarixia radiata Principal parasitoide del psílido asiático de los cítricos, vector del HLB. México: Col. Tepeyac: 2015.

[25] Hernández-Fuentes LM, Urías-López MA, Gómez-Jaimes R, López-Arroyo JI, VelázquezMonreal JJ, Orozco-Santos M. El Huanglongbing y su vector Diaphorina citri en limón persa en Nayarit: Recomendaciones para su manejo. Libro Técnico 3. Santiago Ixcuintla, México: [INIFAP] 2014. ISBN: 978-607-37-0283-6

[26] Kondo T., Tauber C., Guzman-Sarmiento Y.C., Mondragón A., Forero D. A checklist of natural enemies of Diaphorina citri Kuwayama (Hemiptera:Liviidae) in the Department of Valle del Cauca, Colombia and the World. Insecta Mundi. 2015; 0457:1-14.

[27] Sánchez-González JA., Mellín-Rosas MA., Arredondo-Bernal HC, Vizcarra-Valdez NI., GonzálezHernández A., Montesinos-Matías R. Psílido asiático de los cítricos, Diaphorina citri (Hemiptera: Psyllidae). En: Arredondo-Bernal HC, Rodríguez del Bosque LA, editores. Casos de control biológico en México. Vol. 2. Guadalajara, México: Biblioteca Básica de Agricultura. 2015; 339-372.

[28] Kondo T., González F.G., Guzmán Sarmiento, Y. C. Capítulo I. Enemigos naturales de Diaphorina citri. En T. Kondo (Ed.), Protocolo de cría y liberación de Tamarixia radiata Waterston (Hymenoptera: 
Eulophidae). (Corpoica), Mosquera - Colombia: 2017. p23-32

[29] Rodríguez-Palomera M., Cambero-Campos J., Robles-Bermúdez A., Carvajal-Cazola C., EstradaVirgen O. Enemigos Naturales Asociados a Diaphorina citri Kuwayama (Hemiptera: Psyllidae) en Limón Persa (Citrus latifolia Tanaka) en Nayarit, México. Acta Zoológica Mexicana (n.s.): 2012; 28(3): 625-629.

[30] Cortéz Mondaca E., Lugo Angulo N. E., Pérez Márquez J., Apodaca Sánchez, M. A. Primer Reporte de Enemigos Naturales y Parasitismo Sobre Diaphorina citri Kuwayama en Sinaloa, México. Sinaloa - México. Southwest. Entomol. 2010. 35(1). DOI 10.3958/059.035.0113.

[31] González Cárdenas J. C., Castellanos Sturemark I. E., Fucikovsky Zac L. J., López Herrera M., Sánchez Rojas, G. Coccinélidos como potenciales enemigos naturales de Diaphorina citri (Hemiptera: Psyllidae) en un huerto de cítricos en Tuxpan, Veracruz, México. Veracruz - México: Revista Científica UDO Agrícola: 2012; (4): 855-860.

[32] Lozano M., Jasso J. Identificación de enemigos naturales de Diaphorina citri Kuwayama (HEMIPTERA: PSYLLIDAE) en el estado de Yucatán, México. Fitosanidad [Internet]. 2012; 16 (1): 5-11.

[33] Shivankar V.J. Rao, CN. Los psílidos y su gestión. Manejo de plagas en ecosistemas hortícolas. 2010; 16 (1): 1-4.

[34] Cortéz Mondaca E., López Arroyo I. J., Rodríguez Ruíz L., Partida Valenzuela M. P., Pérez Márquez J. Especies de Chrysopidae asociadas a Diaphorina citri Kuwayama en cítricos y capacidad de depredación en Sinaloa, México. Sinaloa - México. Revista mexicana de ciencias agrícolas. 2016; 7(2).

[35] Palomares Pérez M., Ayala Zermeño M. A., Rodríguez Vélez B., de la Cruz Llanas J. D., Sánchez González J. A., Arredonde Bernal H. C., Córdoba Urtiz E. G. Abundancia y depredación de Ceraeochrysa Valida (Neuroptera: Chrysopidae) sobre Diaphorina Citri (Hemiptera: Liviidae) en Colima, México. Colima - México. J Agric Anim Sci. 2016; 3(32); 234-243.

[36] Gómez M., Nava D., Postali J. Life Table of Tamarixia radiata (Himenoptera: Eulophidae) on Diaphorina citri (Hemiptera: Psyllidae) at Different Temperatures. J. Econ. Entomol. 2012; 105(2):338343.

[37] Ortíz Catón M., Alatorre Rosas R., Valdivia Bernal R., Ortiz Catón, A., Medina Torres, R., y Alejo Santiago, G. Efecto de la Temperatura y Humedad Relativa sobre el desarrollo de los Hongos Entomopatógenos. Nayarit - México: Revista Biociencias. 2011; 2(1): 42-53.

[38] Robles G. M. M., Velázquez M. J. J., Orozco S. M., Manzanilla R. M. A., Flores V. R., Arredondo B. H., Archila M. A. B., Núñez C. M. C., Barba R. M., Reyes M. J. G., Rodríguez A. J. I. Bioecología del Psílido
Asiático de los Cítricos Diaphorina citri Kuwayama (Hemiptera: Psyllidae) en limón mexicano en Colima. Resúmenes del VI Simposio Internacional Citrícola. Tecomán, Colima. 2010; 31-73.

[39] Sánchez B. M. Biología, ecología y control de Diaphorina citri Kuwayama (Hemiptera: Psyllidae). Tesis de Doctorado. Colegio de Postgraduados. Montecillo, Texcoco, Edo. de México. 2010.

[40] Miranda S. M., López A. J. I. Fluctuación poblacional del Psílido Asiático de los Cítricos Diaphorina citri Kuwayama (Hemiptera: Psyllidae) y de sus enemigos naturales en Michoacán. Monterrey, Nuevo León, México. 2010. p. 13-18

[41] Rohrig E., Hall D.G., Qureshi J.A., Stansly P.A. Field release in Florida of Diaphorencyrtus aligarhensis (Hymenoptera: Encyrtidae) an endoparasitoid of Diaphorina citri (Homoptera: Psyllidae) from mainland China. Fla. Entomol. 2012; 95: 479-481.

[42] Hall D. G., RichardsonM. L., Ammar E., Halbert S. E. Asian citrus psyllid, Diaphorina citri, vector of citrus Huanglongbing disease. Entomol. Exp. Appl. 2012; 146: 207-223.

[43] Mellín Rosas M. A., Sánchez González J. A., Cruz Ávalos A. M., Montesinos Matías R., Arredondo Bernal H. C. Patogenicidad de Cepas de Hongos Entomopatógenos sobre Diaphorina citri Kuwayama en Condiciones de Laboratorio. México: Southwest. Entomol., 2016; 41(3):791-800

[44] Conant, J., Fadem P. Guía comunitaria para la Salud Ambiental. Berkeley, California, EE. UU.: Hesperian; ISBN: 978-0-942364-59-0. 2011

[45] ONU. Los pesticidas son los responsables de la muerte de 200.000 personas cada año. [Internet]. 2017. Disponible en: https://www.elespectador. com/noticias/medio-ambiente/los-pesticidasson-los-responsables-de-la-muerte-de-200000personas-cada-ano-onu-articulo-683570[11] Gottwald T.R. Current epidemiological understanding of citrus huanglongbing. Annu. Rev. Phytopathol. 2010; 48: 119-139. 